\title{
Adjoint Optimization Method for Head Shape of High- Speed Maglev Train
}

\author{
H. C. $\mathrm{Li}^{1,2,3}$, Z. M. Yang ${ }^{1,2,3}$, L. Zhang ${ }^{1,2,3 \dagger}$ and R. $\mathrm{Li}^{4}$ \\ ${ }^{1}$ Key Laboratory of Traffic Safety on Track of Ministry of Education, Central South University, Changsha, \\ Hunan 410075, China \\ ${ }^{2}$ Joint International Research Laboratory of Key Technology for Rail Traffic Safety, Changsha, \\ Hunan 410075, China \\ ${ }^{3}$ School of Traffic \& Transportation Engineering, Central South University, Changsha, \\ Hunan 410075, China \\ ${ }^{4}$ School of Engineering, Department of Civil Engineering, University of Birmingham, \\ B15 2TT Birmingham, UK
}

†Corresponding Author Email: gszxzhanglei@126.com

(Received September 21 2020; accepted May 18, 2021)

\begin{abstract}
The head shape of a high-speed maglev train was optimized in this study, based on the adjoint method, and the aerodynamic drag of four optimized train models were simulated and compared using different control point generation methods. The effectiveness of using the adjoint method to develop a compressible model for a maglev train was verified. The results show that the adjoint matrix optimization method can quickly and effectively capture the shape characteristics of the train head that are sensitive to aerodynamic resistance. When the design variables of the head are not defined separately, the grid control point set and surface control point set can be used to carry out the adjoint closed-loop optimization of the train head shape, and the exchange control point generation method can be used to perform closed-loop optimization. The results of a numerical simulation show that the optimized train model reduces aerodynamic resistance by approximately $4.8 \%$.
\end{abstract}

Keywords: Maglev; Aerodynamic drag; Adjoint method; Optimization.

\section{NOMENCLATURE}

$\begin{array}{ll}A & \text { reference area } \\ C_{D} & \text { drag coefficient } \\ D & \text { drag force } \\ c_{l} & \text { a constant } \\ d_{k} & \begin{array}{l}\text { displacement vector of the } k \text { th control } \\ \text { point }\end{array} \\ d^{\prime}|X| & \text { displacement of the grid node } \\ F & \text { objective function } \\ f_{b, l}\left(r_{k l}\right) & \begin{array}{l}\text { radial basis function } \\ k\end{array} \\ N & \text { is the number of design variables } \\ R & \text { number of control points } \\ & \text { numerical residual }\end{array}$

\section{INTRODUCTION}

With the continuous development and improvement of high-speed train technology, train running speeds are increasing. Rapid increases in train speed have led to increased drag and energy consumption. Shape

$\begin{array}{ll}r_{k l} & \text { distance between two control points } \\ S_{i} & \text { search direction } \\ X & \text { coordinate vector of the grid node } \\ x & \text { design variable } \\ x_{l} & \text { coordinate vector of the control point. } \\ x_{k}^{S} s \text { th } & \text { coordinate of the } k \text { th node in the mesh } \\ \alpha & \text { constant vector } \\ \alpha_{i} & \text { search step length } \\ \lambda_{l} & \text { expansion coefficient } \\ \psi^{T} & \text { adjoint solution variable }\end{array}$

optimization is an important method of drag reduction. Aerodynamic shape optimization, which aims to improve the aerodynamic performance of high-speed trains and solve some aerodynamic problems, plays an important role in the design of high-speed trains. The aerodynamic problems of 
high-speed trains are closely related to the air flow around the vehicles. In the past decade, several researchers have carried out extensive research on high-speed trains and maglev air flow characteristics under different scenarios (e.g., Hemida and Krajnovic 2010; Baker 2010; Krajnovic et al. 2012; Huang et al. 2019; Jia and Mei 2018). Once the flow field structure has been measured, it is possible to carry out targeted optimization. Genetic algorithms, particle swarm optimization, and other optimization methods can extract valuable information from existing data and solve optimization problems through specific strategies. Vytla (2011) carried out single-objective optimization for multiple design variables in the context of an open-air environment using geometric parameterization and a genetic algorithm. Yao et al. (2014) used a genetic algorithm to improve the safety and comfort of train operation with aerodynamic lift of the tail car and streamlined head volume as objectives. Munoz-Paniagua et al. (2014) used a genetic algorithm to optimize the train head shape to reduce the maximum pressure gradient at the tunnel entrance. Zhang et al. (2017) took the total aerodynamic resistance and tail car aerodynamic lift as optimization objectives and carried out multi-objective aerodynamic optimization using a non-dominated sorting genetic algorithm (NSGA-II) and Kriging model, which improved the aerodynamic performance of trains in environments with and without cross winds. Li et al. $(2016,2020)$ used a free-deformation method and non-dominated sorting genetic algorithm to select multiple geometric parameters for drag reduction and multi-objective optimization of high-speed trains. The computational complexity of the optimization depends on the number of design variables that parameterize the best candidate features. The realizable improvement depends on the choice of geometric parameters (Othmer and Grahs 2005). Therefore, the calculation of the variable values can be carried out flexibly and reliably. This strategy becomes more critical as the complexity of high-speed train flow simulation increases.

The adjoint optimization method is based on the objective function gradient. Its sensitivity is independent of the number of design variables; therefore, it can save time and reduce costs. Each point on the optimization surface can be regarded as a design variable. The adjoint method eliminates constraints imposed by the dimension of geometric design and can calculate all gradient components. The cost is independent of the number of gradient components. Design and optimization using adjoint methods is a topic of interest in various engineering fields, such as aviation (Nejati and Mazaheri 2017; Bobrowski et al. 2017), automotive engineering (Othmer 2014; Han et al. 2016), and thermal and hydraulic turbine design (Mueller et al. 2012). In fluid mechanics, Pironeau (1974) was the first to propose the adjoint design equation. In practical applications of computational fluid dynamics simulation optimization, the gradient-based method and adjoint method are combined (Jameson 1988; Jameson and Martinelli 1998; Giles and Pierce 1997) to calculate the sensitivity derivative, that is, the gradient of the objective function relative to the design variable. In aerospace engineering, Lei et al. (2019) used the adjoint method to study the integrated aerodynamic design of aircraft and power engines to optimize the layout of wing engines while reducing overall drag. Kapsoulis et al. (2018) used the adjoint method and a genetic algorithm to carry out section optimization of an aircraft airfoil and obtained optimized results for two kinds of wing airfoils based on maximum lift and minimum torque. Kun et al. (2018) used a discrete adjoint method and two curve control methods to generate two transonic airfoil optimization schemes to reduce drag under constraints of wing cross-sectional area and lift. Kungurtsev and Juniper (2019) used a gradientbased adjoint method to optimize the shape of a droptype ink-jet printhead to suppress residual reverberation and vibration without increasing the pressure drop required to drive steady flow. Shape optimization using the adjoint method to reduce total pressure loss and increase the tumble moment has also attracted attention (Verma et al. 2018; Kubota et al. 2016). In vehicle shape design, sensitive components are selected based on a sensitivity analysis based on gradients, and continuous adjoint methods and discrete adjoint methods are used to optimize vehicle aerodynamic shapes to improve aerodynamic resistance and lateral torque under wind measurement conditions (Papoutisiskiachagias and Giannakoglou 2016; Papoutisiskiachagias et al. 2019). Munoz Paniagua et al. (2015) used the adjoint method to optimize the shape of high-speed train heads, demonstrating the effectiveness of the method. When the number of solver calls is low, the aerodynamic resistance is also significantly reduced. Zhang (2017) used a set of surface control points to optimize the head shapes of high-speed trains, reducing the aerodynamic resistance in open-line environments.

The purpose of this study was to prove the effectiveness of the adjoint method in the optimization of maglev train head shapes. In adjoint optimization with aerodynamic drag as the objective, the optimization results of two methods for generating the optimal control point set of the head shape were compared. The effectiveness of the single-objective adjoint optimization method for maglev train head shape was evaluated using a flow field analysis optimization model.

\section{METhOD AND Formulation OF THE PROBLEM}

The objective of this study was to optimize the head shape of a high-speed maglev train by reducing the aerodynamic drag coefficient when the train faces into the wind. This single-objective optimization problem can be defined as follows:

$$
\begin{array}{lll}
\text { Minimize } & F(x) & \\
\text { subject to } & g_{j}(x)=0 & j=1 \ldots m \\
& h_{p}(x)=0 & l=1 \ldots m . \\
& x_{k}^{l} \leq x_{k}^{l} \leq x_{k}^{l} & k=1 \ldots m
\end{array}
$$




$$
x_{i+1}=x_{i}+\alpha_{i}
$$

where $\alpha_{i}$ is the search step length in the search direction $S_{i}$. Based on the definition of the search direction, different optimization algorithms can be formed. If the search step size is calculated based on the value of the objective function, a zero-order algorithm, such as a genetic algorithm, can be used; if the search step size is calculated based on the first derivative of the objective function, a gradient algorithm can be used.

\subsection{Discrete joint formulation}

The simplest way to define the search direction is $S_{i}=-\nabla F\left(x_{i}\right)$, and the following finite difference scheme can be used for gradient calculation:

$$
\frac{\mathrm{d} F}{\mathrm{~d} x_{k}}=\frac{F\left(x_{k}+\Delta x_{k}\right)-F\left(x_{k}\right)}{\Delta x_{k}}, k=1,2, \cdots, K
$$

where $F$ is the objective function, and $k$ is the number of design variables.

The adjoint method can be used to reduce the computational complexity of the objective function relative to the gradient $\mathrm{d} F f \mathrm{~d} x_{k}$ of the optimal design variables. The main feature of the adjoint method is that the calculation of the gradient of the optimal design variables by the optimization objective function is independent of the number of optimal design variables. In this way, it can significantly reduce the time consumed during the calculation of the optimal design variable gradient of the objective function (Papadimitriou and Giannakogluu 2008).

When a series of design variables $x$ are given to optimize the train head shape, the objective function $F$ is not only related to the head shape design variable $x$ but also to the flow field variable $u$ on the grid node. Therefore, the gradient of the objective function relative to the design variable can be expressed as follows:

$\frac{\mathrm{d} F}{\mathrm{~d} x}=\frac{\mathrm{d} F}{\mathrm{~d} u} \frac{\mathrm{d} u}{\mathrm{~d} \chi}+\frac{\partial F}{\partial x}$

The constraint conditions are satisfied:

$\frac{\mathrm{d} R}{\mathrm{~d} x}=\frac{\partial R}{\partial u} \frac{\mathrm{d} u}{\mathrm{~d} x}+\frac{\partial R}{\partial x}=0$

where $R$ is the numerical residual. According to formula (5), it can be concluded that:

$\frac{\mathrm{d} u}{\mathrm{~d} x}=-\left(\frac{\partial R}{\partial u}\right)^{-1} \frac{\partial R}{\partial x}$

Substituting formula (6) into equation (4), we can get the following results:

$\frac{\mathrm{d} F}{\mathrm{~d} x}=-\frac{\partial F}{\partial u}\left(\frac{\partial R}{\partial u}\right)^{-1} \frac{\partial R}{\partial x}+\frac{\partial F}{\partial x}$

By introducing the multiplier $\psi^{T}$, the gradient of the objective function relative to the design variable can be written as follows:

$\frac{\mathrm{d} F}{\mathrm{~d} x}=-\psi^{T} \frac{\partial R}{\partial x}+\frac{\partial F}{\partial x}$

where $\psi^{T}$ is the adjoint solution variable, the following equation is satisfied:

$\frac{\partial F}{\partial u}=\psi^{T} \frac{\partial R}{\partial u}$

Equation (9) is the adjoint equation, from which we can see that the adjoint equation only needs to solve the current flow field variable $U$, and it has nothing to do with the design variable $\mathrm{x}$. To solve the gradient of the objective function relative to the design variables, it is not necessary to do one numerical calculation for each design variable but only need to solve the initial flow field and the adjoint flow field. This can significantly save the time consumed during the optimization of the design.

Figure 1 shows a workflow with optimization. In the fluid simulation software, the overall framework of shape optimization based on gradient continuous adjoint method is realized with the goal of adjoint flow optimization. The modules used in each optimization cycle include: 1. Solution of original flow equation; 2. Solution of objective function; 3 . Solution of adjoint equation; 4. Gradient calculation; 5. Updating design variables; and 6. Geometric displacement and mesh deformation. After optimization, the optimization results are verified, and it is observed that the results are in line with the requirements.

\subsection{Surface deformation and mesh deformation}

$d F=\frac{\partial F}{\partial x_{k}^{s}} d x_{k}^{s}$

Among them, the deformation node is the node of the surface mesh. $x_{k}^{s}$ is the $s$ th coordinate of the $k$ th node in the mesh ( $x_{k}$ is the $k$ th node). Then

$d x_{k}^{s}=c \frac{\partial F}{\partial x_{k}^{s}}$

provides the maximum adjustment of $F$ for given $L^{2}$ norm of $d x_{k}^{s}, \quad c$ is an arbitrary scale factor. It should be noted that the use of formula (10) to calculate $\partial F / \partial x_{k}^{s}$ in some areas may result in many inflection points on the target surface. Therefore, to ensure the continuity of the surface, it is necessary to use the mesh deformation tool to smooth the surface sensitive field and internal mesh.

In this study, the radial basis function-based mesh deformation method is used to deform the maglev train head. This method is used for aerodynamic optimization of the head shape, which can realize the smooth deformation of the head shape and avoid the repeated generation of the mesh in the optimization 


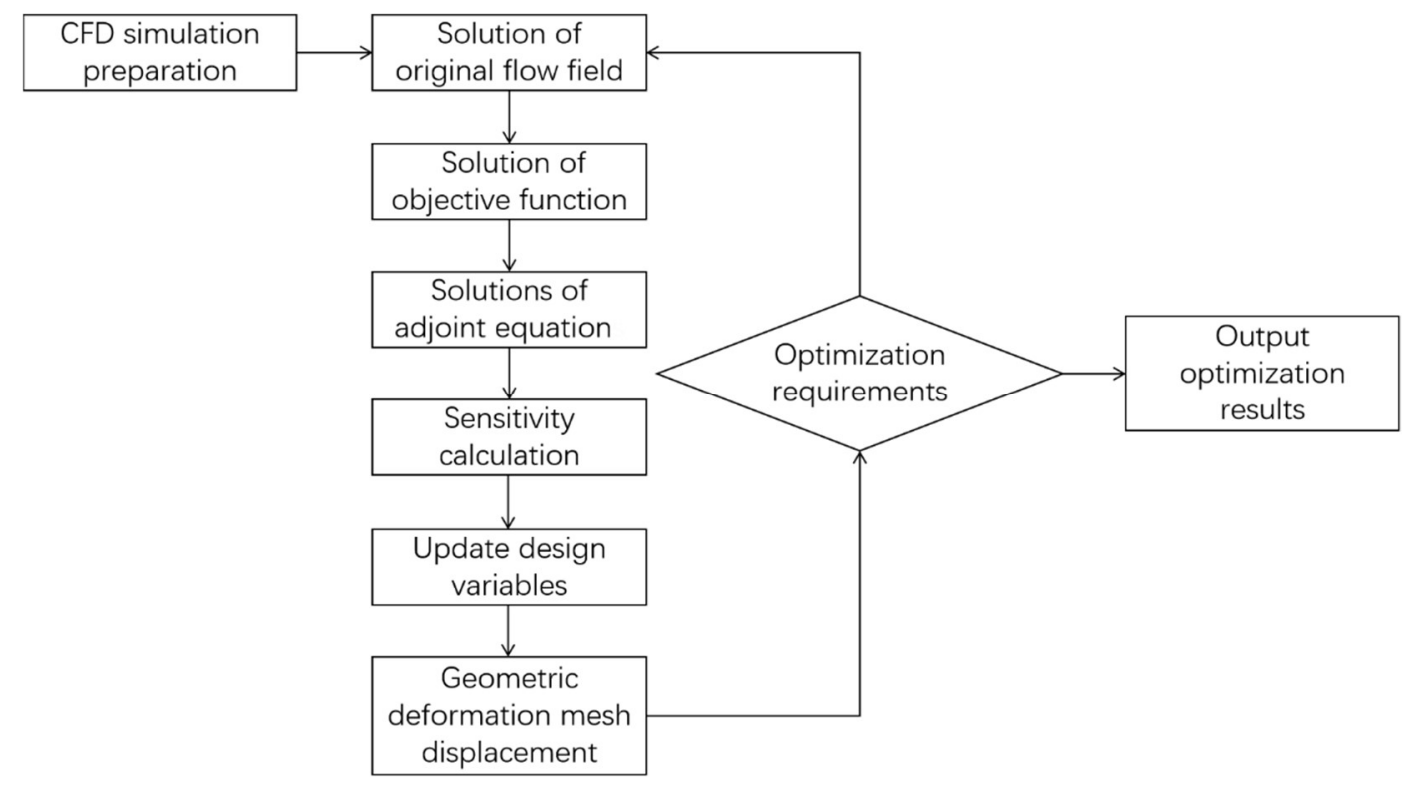

Fig. 1. Adjoint optimization flow chart.

process, to improve the efficiency of the optimization design. A series of control points are arranged around the train head to control the deformation of the grid. Each control point is related to the displacement of its surrounding mesh nodes. Based on the interpolation theory, a mesh deformation interpolation field can be generated for a given control point displacement. To generate the interpolation field, the control point displacement is expanded as follows:

$d_{k}=\sum_{=1}^{N} f_{b, l}\left(r_{k l}\right) \lambda_{l}+\alpha$

where, $d_{k}$ is the displacement vector of the $k$ th control point, $N$ is the number of control points, $\lambda_{l}$ is the expansion coefficient, $\alpha$ is the constant vector, and $f_{b, l}\left(r_{k l}\right)$ is the radial basis function, which are expressed as follows:

$$
f_{b, l}\left(r_{k l}\right)=\sqrt{r_{k l}^{2}+c_{l}^{2}}
$$

$c_{l}$ is a constant, which is set to 0 in this study, and $r_{k l}$ is the distance between two control points:

$$
r_{k l}=\left|x_{k}-x_{r}\right|
$$

The expansion coefficient, $\lambda_{l}$, satisfies the constraint conditions

$$
\sum_{l=1}^{N} \lambda_{l}=0
$$

When the displacements of each control point are known, $\lambda_{l}$ and constant vector $\alpha$ can be obtained simultaneously using Eqs. (12) and (15), and the displacement interpolation of grid nodes can be obtained as follows:
$\mathrm{d}^{\prime}|X|=\sum_{l=1}^{N} f_{b, l}(r) \lambda_{l}+\alpha$

$\mathrm{d}^{\prime}|X|$ is the displacement of the grid node, $f_{b, l}(r)=\sqrt{r^{2}+c^{2}}, \quad r=\left|X-x_{l}\right|, \quad X \quad$ is the coordinate vector of the grid node, and $x_{l}$ is the coordinate vector of the control point.

Based on the calculation of initial and adjoint flow fields, the sensitivity data of the objective function to the coordinates of control points are obtained.

The displacement $d$ of each control point can be obtained by the Eq. (11).

$$
\mathrm{d}\left(x_{k}^{s}\right)=c \frac{\partial F}{\partial x_{k}^{s}}
$$

From the displacement of control points, the displacements of each grid node can be obtained by Eqs. (12), (15), and (16), to realize the mesh deformation in the calculation area.

\section{OPTIMIZED APPLICATION}

The preprocessing of the numerical simulation and the associated optimization cycle calculation are carried out in star-ccm+. Closed loop and iterative solution are allowed in the software. In the optimization process, the convergence of the simulation is affected by the quality of the deformed mesh, and we observe that the mesh quality will gradually decrease with each iteration. Therefore, it is necessary to check the mesh quality after each optimization cycle and repair the grid with poor quality, to ensure the stability of grid quality.

\subsection{Optimization model}

The three-car body model investigated in this study 
is based on the maglev train model of Shanghai maglev line, which is in commercial operation. This study focuses on the aerodynamic drag coefficient change of high-speed maglev train caused by the deformation in head shape optimization. Therefore, the body part of the proportional model is selected, including the proportional streamline and the proportional carriage. It is $3.7 \mathrm{~m}$ wide, $3 \mathrm{~m}$ high, and $79 \mathrm{~m}$ long. The distance between the body of maglev train and the maglev track is defined by the bottom edge of the model head car. In the optimization work, it is necessary to ensure that the maximum range of optimization deformation conforms to the relevant specifications of a maglev train.

To obtain the resistance coefficient of the car body, a calculation domain, as shown in Fig. 2, is established. The entrance is $60 \mathrm{~m}$ away from the head car, and the exit is $200 \mathrm{~m}$ away from the tail car. The width and height of the calculation domain are both $80 \mathrm{~m}$. The car body model is arranged in the middle. The inlet and outlet boundaries are free flow, and the velocity is Mach 0.49 . The other boundaries are symmetrical. The Reynolds number based on entrance speed and train height is $3.4 \times 10^{7}$.

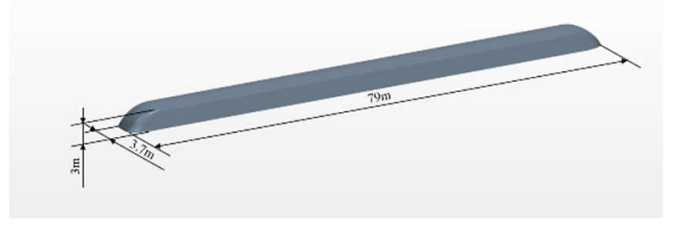

(a) The train model

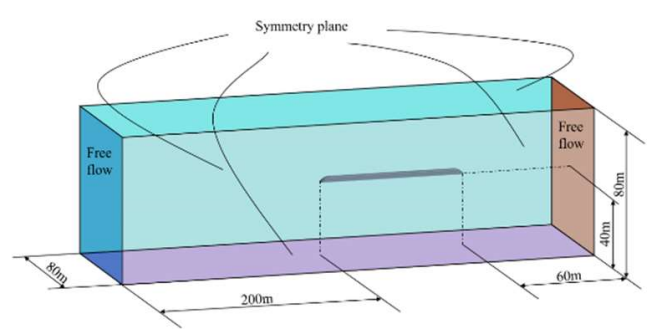

(b) Computational domain

Fig. 2. Car body model and computational domain.

\subsection{Turbulence Model and Numerical Set-up}

In the solver of star-CCM +, the adjoint optimization only supports the steady turbulence model. Therefore, the compressible steady K - $\omega$ (RANS) turbulence model is used for fluid simulation in this study. Previous studies (Papoutsis-Kiachagias et al.2019) have demonstrated the effectiveness of RANS-based turbulence model for adjoint optimization. To establish a reliable flow field, the grid is divided as shown in the Fig. 3. Ten prismatic layers are used, and the parts near the train body are properly encrypted. The mesh in dependence analysis shows that the final number of mesh is 8.34 million. The value of $\mathrm{Y}+$ is $25 \sim 250$.

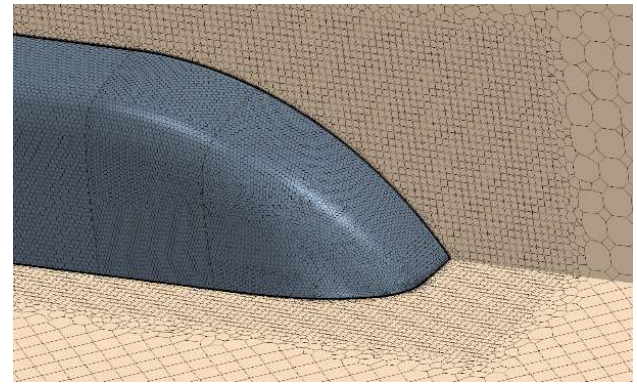

(a) Computational mesh

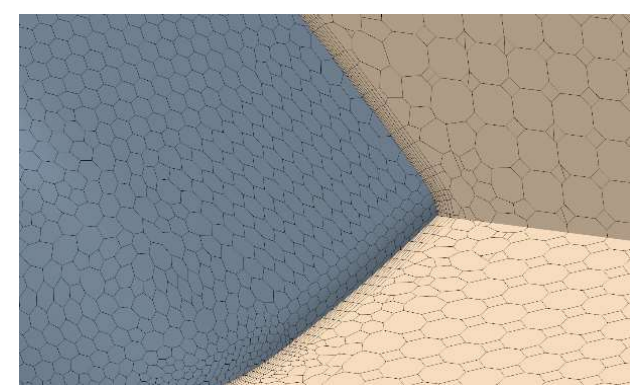

(b) Prism layer mesh

Fig. 3. Computational mesh.

\subsection{Sensitivity Analysis}

Before starting the optimization cycle, the sensitivity analysis of the target model is established. A sensitivity map is established based on the aerodynamic drag gradient of the car body model. We can observe that the sensitivity of most areas of the car body is within \pm 40 . However, the tip of the nose had a higher value. The red area of the model with reduced resistance represents the outward displacement of the model. Therefore, to reduce the aerodynamic resistance of the car body, the streamline of the car body should be selected, especially the deformation of the model at the nose.

\subsection{Optimization Settings}

In this study, grid control set points and surface set control points are used to discrete adjoint optimization of the model. After the first round of five optimization cycles, two optimization models were obtained. After the first round of optimization, another method was used to control the generation of assembly points, and the second round of optimization cycle was carried out three times. Four optimization results were obtained. They are named as A (grid set), B (surface set), C (grid set-surface set), D (surface set-grid set), and the original model is $\mathrm{O}$. After the four optimized head shapes were obtained, the two methods of generating set control points were compared. RANS equation turbulence model and discrete adjoint optimization method were used to simulate and optimize the optimization process. The first round of optimization is five optimization cycles, and the second round is three optimization cycles. The main consideration is that the grid quality has been significantly reduced because of the increase in the number of closed-loop 
optimization cycles. At the same time, the optimization effect was not sufficiently clear, and even the inflection point of aerodynamic resistance rising trend appeared. Therefore, the optimization results $(\mathrm{A}, \mathrm{B}, \mathrm{C}, \mathrm{D})$ with the best air performance and better grid quality were selected as the output results.

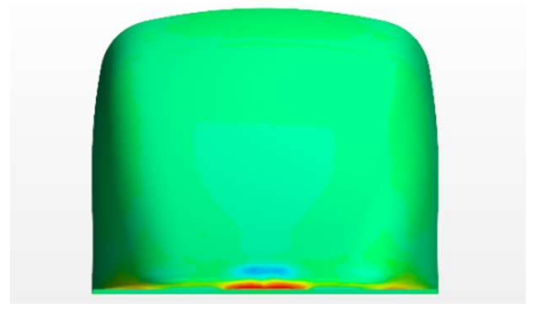

(a) front

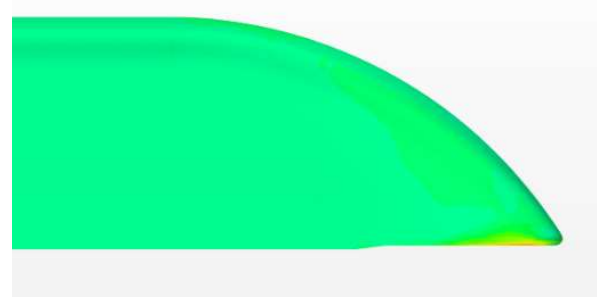

(b) side

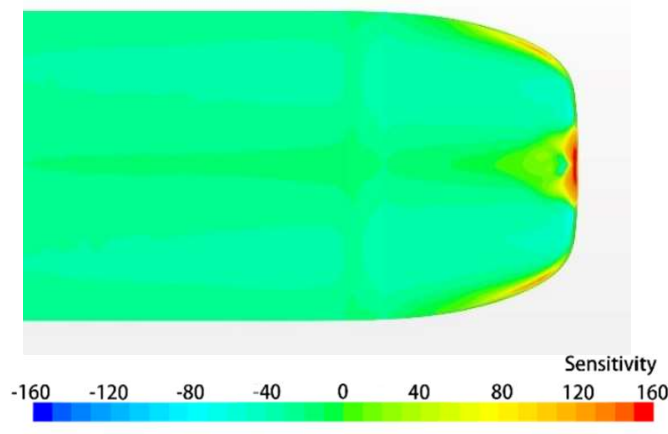

(c) bottom

Fig. 4. Sensitivity diagram of car body: the blue area is negative, which means that the outward displacement of the surface will increase the resistance; the red area is positive, which means that the outward displacement of the surface will reduce the resistance.

The generation of grid set control points is shown in Fig. 3. Based on the size of the train head, the size of the control box is $5.5 \mathrm{~m} * 3.9 \mathrm{~m} * 3.4 \mathrm{~m}$, and the control point is $10 * 8 * 6$. Grid set control points are generated equally. The generation of surface collective control points is shown in Fig. 5. Based on the shape of the deformable zone of train nose, the surface collective control points with uniform point distance of $0.3 \mathrm{~m}$ are distributed at a distance of 0.3 $\mathrm{m}$ away from the surface.

In the adjoint solver, the mesh deformation algorithm was the steepest descent method. To ensure the fine adjustment of the head shape and ensure that the original streamline length does not change too much, we set the deformation step size as $1.5 \times 10^{-5}$.

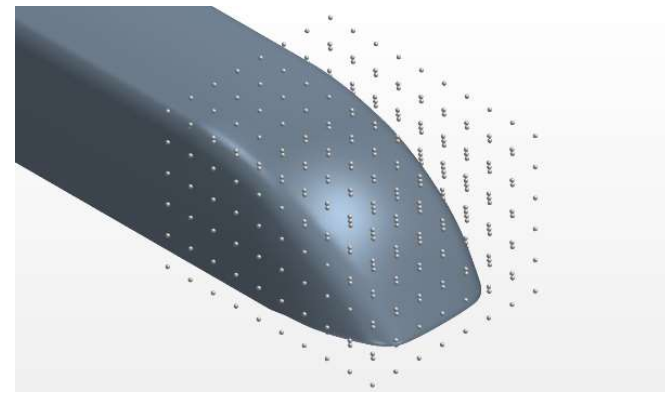

(a) Grid set control point

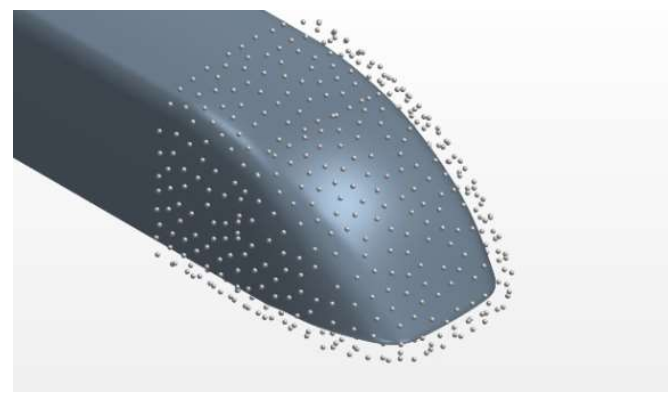

(b) Surface set control point

Fig. 5. Two sets of control points.

\subsection{Optimization Results}

As shown in Fig. 6, the four types of Maglev heads have different deformations. At the end of the first optimization cycle, the nose tip of model A had obvious lifting deformations. While the nose tip had obvious inward deformation at the excessive streamline behind the nose tip, the length of the nose tip did not changed significantly, and the cab shoulder had also contracted inward. Regarding model B, the nose tip protruded and thinned forward, height did not change significantly, and cab shoulder contracted inward. After the initial optimization of the grid set control points, the surface collective control points were optimized for the second time, and the optimization result $\mathrm{C}$ showed that the nose tip height was further raised compared with $\mathrm{A}$, the nose tip length was reduced compared with $\mathrm{O}$ and $\mathrm{A}$, and the back of the nose tip and cab shoulder were further contracted inward. After the initial optimization of surface set control points, it can be observed from optimization result $\mathrm{D}$ that the height of the nose tip is slightly increased compared with $\mathrm{O}$ and $\mathrm{B}$, and the length of the nose tip is not significantly different.

\section{VERIFICATION APPLICATION}

\subsection{Validation Model}

The aerodynamic performance of the four optimization models is tested under open-air 


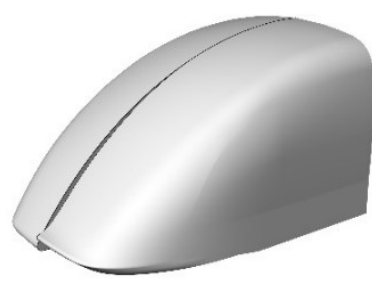

(a) Grid set control point model A

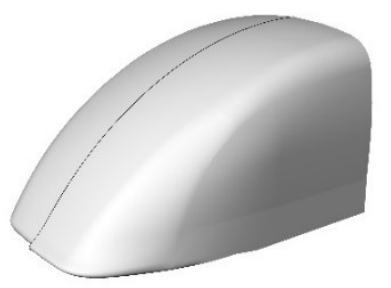

(b) Surface set control point model B

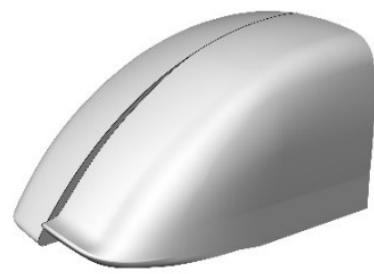

(c) Grid-Surface set control point model C

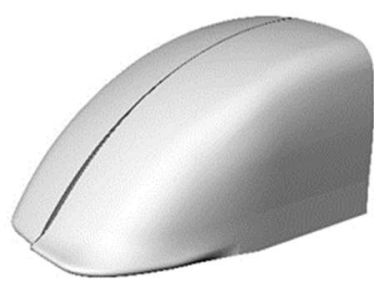

(d) Surface-Grid set control point model D

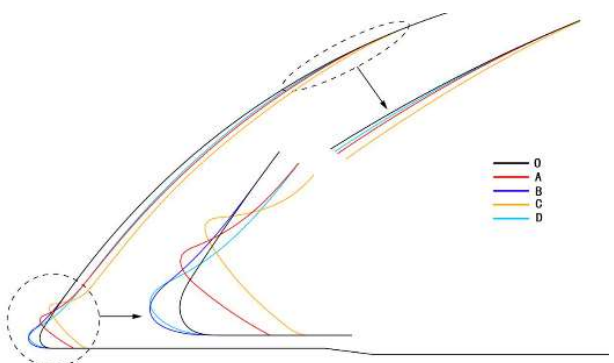

(e) Original profile and optimized profile

Fig. 6. Optimization results: the inner side is the original model; the outer side is the optimized model. conditions, and it is investigated whether the optimization of body streamline is effective for the overall aerodynamic resistance of a high-speed maglev train. The tr- 08 three car equal ratio model is used in this study, which is $79 \mathrm{~m}$ long, $3.7 \mathrm{~m}$ wide, and $4.1 \mathrm{~m}$ high. The track height of the maglev train is $2.1 \mathrm{~m}$. The calculation domain in front of the vehicle is $80 \mathrm{~m}$ in length, $140 \mathrm{~m}$ in width, and $40 \mathrm{~m}$ in height in the rear. The model is arranged in the middle and conforms to the national standard TB / T 3503.4-2018. The inlet and outlet of the flow field are free flow, velocity is Mach 0.49 , ground and orbit are moving walls, and boundary of other computational domains are symmetric surfaces. The compressible steady K- $\omega$ (RANS) turbulence model is used for fluid simulation. The narrow gap between track and car body uses a structural grid, and other parts use an unstructured grid. The total number of computational domain grids is 66.26 million.

\subsection{Numerical Simulation Results}

The drag coefficient calculated by numerical simulation of vehicle model shows that both gridset control points and surface set control points have certain drag reduction effect. The aerodynamic drag of grid set optimization A is reduced by $3.2 \%$ and that of surface set optimization B is reduced by $3.7 \%$. At the end of the second optimization cycle, the drag reduction effect of optimization model $\mathrm{B}$ and $\mathrm{D}$ is further than that of the previous model, but the reduction range is obviously less than that of the first optimization cycle. The aerodynamic drag of grid surface ensemble optimization B is reduced by $3.9 \%$, and that of surface grid ensemble optimization $\mathrm{D}$ is reduced by $4.8 \%$. In general, both the grid set and the surface set control point optimizations have certain drag reduction effect. The drag reduction effect of the second optimization cycle is not as obvious as that of the first optimization cycle.

$$
C_{D}=\frac{D}{\frac{1}{2} \rho V^{2} A}
$$

where $D$ is the drag force on the train, $\rho$ is the air density $\left(1.225 \mathrm{~kg} / \mathrm{m}^{3}\right)$, and $A$ is chosen as $10.9 \mathrm{~m}^{2}$.

Table 1 Comparison of aerodynamic drag coefficients of optimization models.

\begin{tabular}{|c|c|c|}
\hline Model & $C_{D}$ & $C_{D} \%$ \\
\hline $\mathrm{O}$ & 0.3417 & - \\
\hline $\mathrm{A}$ & 0.3306 & -3.248 \\
\hline $\mathrm{B}$ & 0.3280 & -3.922 \\
\hline $\mathrm{C}$ & 0.3289 & -3.746 \\
\hline $\mathrm{D}$ & 0.3252 & -4.829 \\
\hline
\end{tabular}

The deformation zones of the five simulation models were analyzed separately. The drag coefficients of the five groups of models shows that the drag coefficient of the original model $\mathrm{O}$ is the largest, while the drag coefficient of the optimized model A 


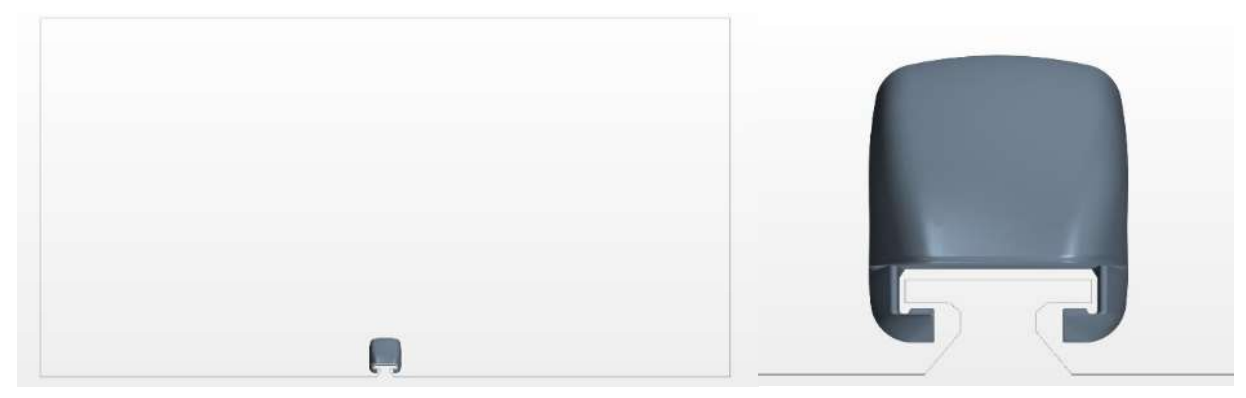

Fig. 7. Computational domain of validation model.

Table 2 Pressure in deformation zone of optimization model.

\begin{tabular}{|c|c|c|c|c|c|c|}
\hline \multirow{2}{*}{ Model } & $\begin{array}{c}\text { Pressure } \\
\mathrm{drag}\end{array}$ & $\begin{array}{c}\text { Shear } \\
\text { resistance }\end{array}$ & & $\begin{array}{c}\text { Drag } \\
\text { coefficients }\end{array}$ & \\
\cline { 2 - 7 } & $\mathrm{Pa}$ & $\%$ & $\mathrm{~Pa}$ & $\%$ & & $\%$ \\
\hline $\mathrm{O}$ & $1.1800 \times 10^{4}$ & & $1.3179 \times 10^{3}$ & & 0.0711 & \\
\hline $\mathrm{A}$ & $1.1491 \times 10^{4}$ & -2.619 & $1.3053 \times 10^{3}$ & -0.956 & 0.0690 & -2.954 \\
\hline $\mathrm{B}$ & $1.0866 \times 10^{4}$ & -7.915 & $1.3779 \times 10^{3}$ & 4.552 & 0.0660 & -7.173 \\
\hline $\mathrm{C}$ & $1.1079 \times 10^{4}$ & -6.110 & $1.3739 \times 10^{3}$ & 4.249 & 0.0671 & -5.626 \\
\hline $\mathrm{D}$ & $1.0012 \times 10^{4}$ & -15.152 & $1.3575 \times 10^{3}$ & 3.005 & 0.0613 & -13.783 \\
\hline
\end{tabular}

and $\mathrm{C}$ is significantly lower than that of the original model. Compared with $\mathrm{A}$ and $\mathrm{C}$, the drag coefficient of the optimization models $\mathrm{B}$ and $\mathrm{D}$ decreased to varying degrees. The drag coefficient in the deformation zone of model D decreases more obviously than that in model B. Compared with model $\mathrm{O}$, the pressure drag of the four optimization models decreased in varying degrees, and the drop in model $\mathrm{D}$ was the most obvious. In terms of shear resistance, the shear resistance of model A decreased by $0.956 \%$, and the shear resistance of other models increased in varying degrees. The decrease of aerodynamic drag of the optimized model is related to the decrease of pressure drag in the optimized deformation region.

\subsection{Flow Field Analysis of Validation Model}

\subsubsection{Surface Pressure}

The surface pressure diagram of the original model was compared with that of the four optimized models on the left and right in Fig. 8. Because the nose tip of model A rises to a certain extent, the positive pressure area of model $\mathrm{A}$ is smaller than that of model O. Based on model A, the nose tip of model C was further raised, resulting in the separation of the positive pressure region at the nasal tip, and the maximum value of the positive pressure area appeared in the lower part of the nasal tip. Meanwhile, the negative pressure area of the cab shoulder is deeper than that of the original model. The positive pressure region of model $\mathrm{B}$ is smaller than that of model O. Based on model B, the positive pressure region of model D is further reduced, which has a significant downward trend compared with the positive pressure region of the nose tip near the surface of the original model. With the optimization, the high-pressure area near the stagnation point can be reduced by the deformation of the deformation region to reduce the aerodynamic drag.

\subsubsection{Pressure Diagram of Symmetry} Plane

Figure 9 shows the pressure distribution on the symmetry plane of the original model and the four optimized models. In the figure, we can see that the positive pressure area in front of the nose tip of the four optimization results is smaller than that of the original model. The nose tip of model A rises to a certain extent, leading to a certain reduction in the positive pressure area compared with the original model, primarily due to the reduction of air mass volume in the high-pressure area. The nose tip of model $\mathrm{C}$ rises further based on $\mathrm{A}$, which results in the separation of positive pressure area, and the maximum value of positive pressure area appears in the lower part of nasal tip. Compared with the original model, the positive pressure area of the nose tip of model B is marginally reduced, while other differences are not significant. Compared with the original model and model $\mathrm{B}$, the positive pressure area of model $\mathrm{D}$ is significantly smaller than that of the original model and model $\mathrm{B}$. The positive pressure area of model $\mathrm{D}$ is the smallest among the four optimization models, and the high-pressure part is also the smallest.

\subsubsection{Streamline}

Figure 10 shows the streamline diagram of the original model and the four optimization models. In the figure, we can see that the near surface streamline of the four optimization models is different from that of the original model. In general, the air velocity at the nose tip of the four models is significantly faster than before. This is consistent with the change of the 


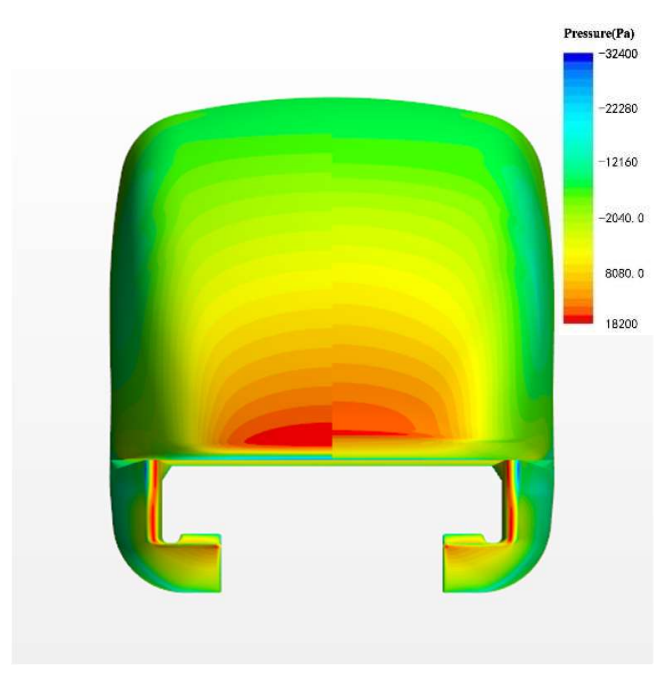

(1) O-A

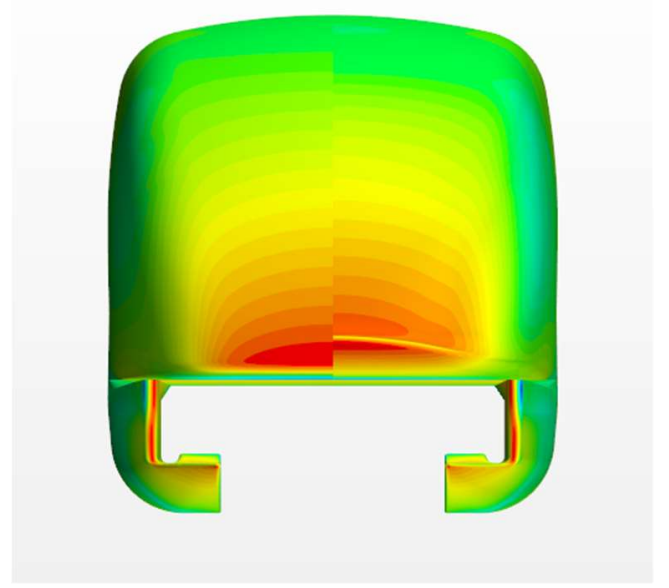

(3) $\mathrm{O}-\mathrm{C}$

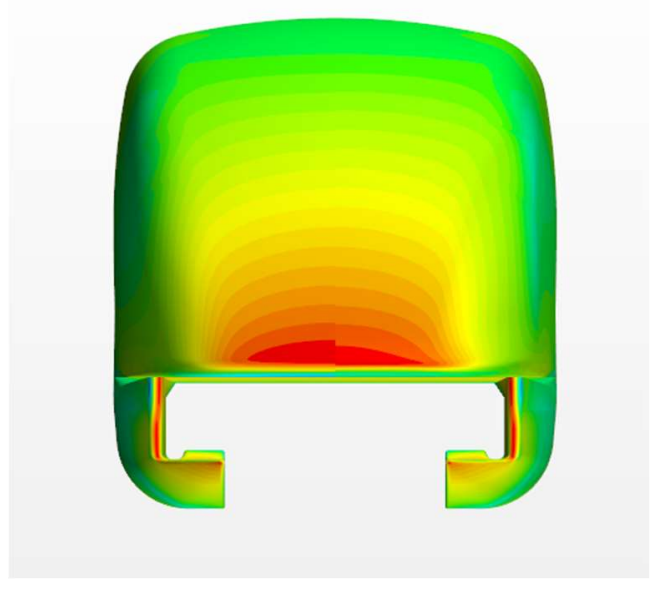

(2) O-B

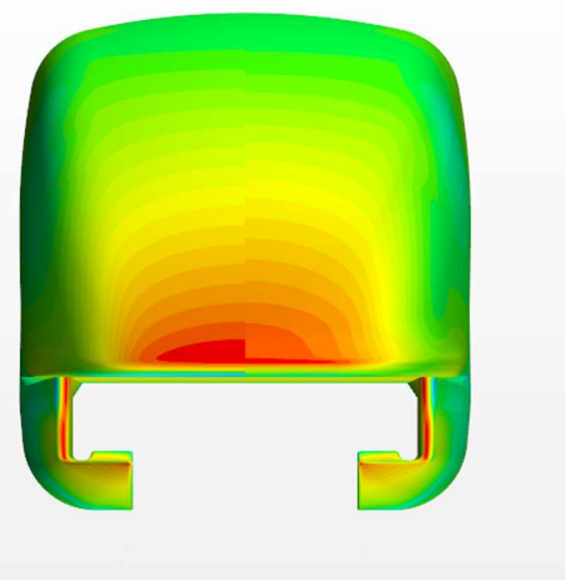

(4) O-D

Fig. 8. Optimize model surface pressure.

pressure distribution of the head carriage of the five models, and the air velocity at the nose tip becomes faster, which reduces the positive pressure area caused by air stagnation at the nose tip. At the same time, the streamline of the cab shoulder shows that the optimization model is faster than the original model when air flows through the cab shoulder.

For model A and C, the rise in the height of the nose tip increases the air separated to the lower part of the nose tip, increases the air velocity under the nose tip, and decreases the positive pressure at the nasal tip. At the same time, the contraction of the cab shoulder improves the flow of the air passing through both sides of the cab, which reduces the aerodynamic resistance at the nose tip. For models $\mathrm{B}$ and $\mathrm{D}$, the nose tip length increases, nose tip width decreases, and cab shoulder shrinks, which accelerates the air velocity in the streamline part of the front car and reduces the aerodynamic resistance at the nose tip.

\section{CONCLUSION}

In this study, the discrete adjoint optimization method and two control sets are used to optimize the maglev car body model, and the maglev equal ratio model is used to verify and analyze the optimization effect. Through the analysis of geometry and flow field, the following conclusions are obtained.

1. In this study, four optimization models are obtained by using the adjoint optimization method. Among them, the nose tips of models $\mathrm{A}$ and $\mathrm{C}$ are raised to a certain extent, and the nasal tips of models $\mathrm{B}$ and $\mathrm{D}$ are elongated to a certain extent, which leads to the reduction in the high-pressure area of the nasal tip stagnation point. The results show that the cross section of the cab shoulder of the four optimization models has been marginally reduced, which is consistent with the aerodynamic trend that the thinner the nose tip, the smaller the aerodynamic resistance. 
H. C. Li et al. / JAFM, Vol. 14, No. 6, pp. 1839-1850, 2021.

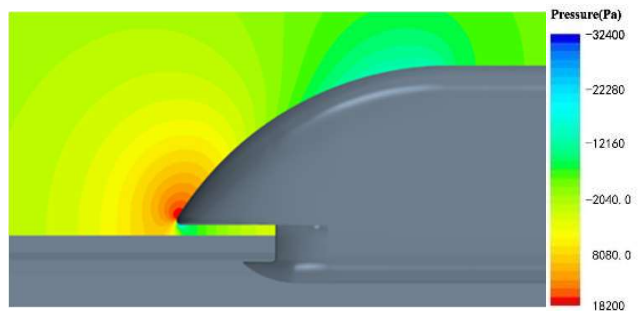

(1) $\mathrm{O}$

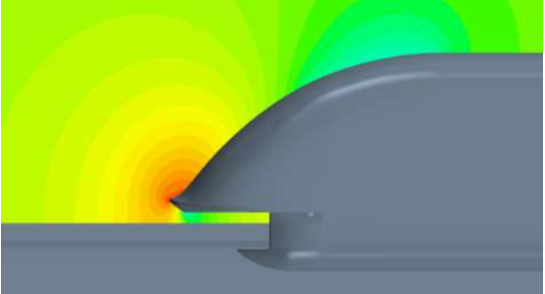

(2) $\mathrm{A}$

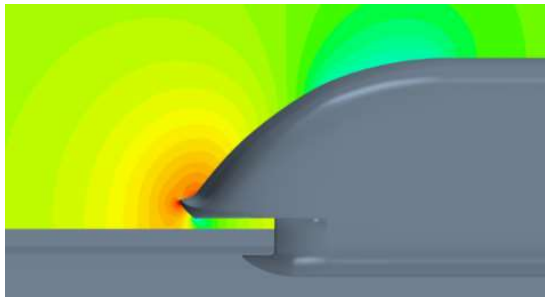

(5) $\mathrm{C}$

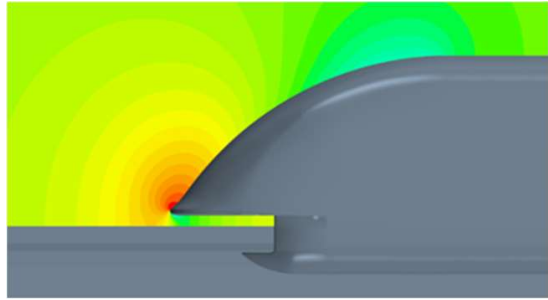

(3) $\mathrm{B}$

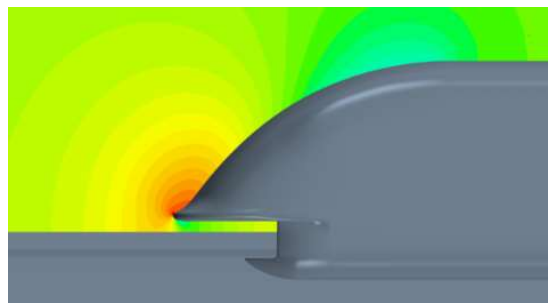

(4) $\mathrm{D}$

Fig. 9. Pressure diagram of symmetry plane of optimization model.

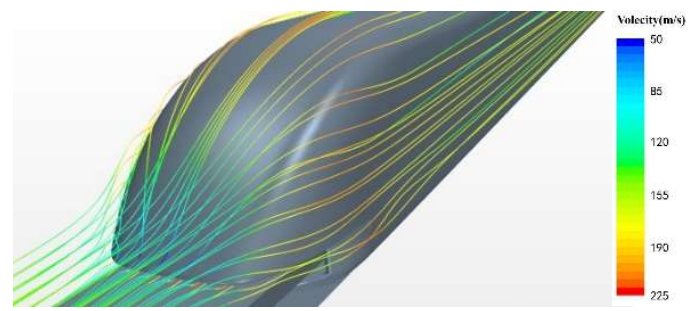

(1) $\mathrm{O}$

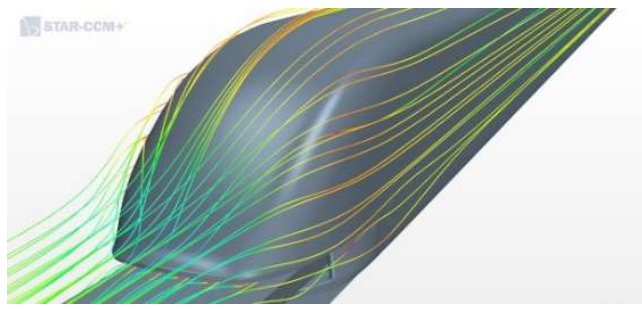

(2) A

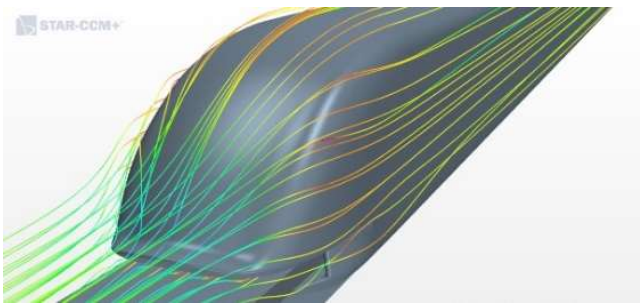

(4) $\mathrm{C}$

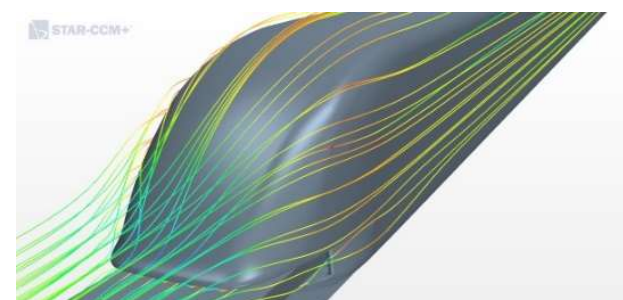

(3) $\mathrm{B}$

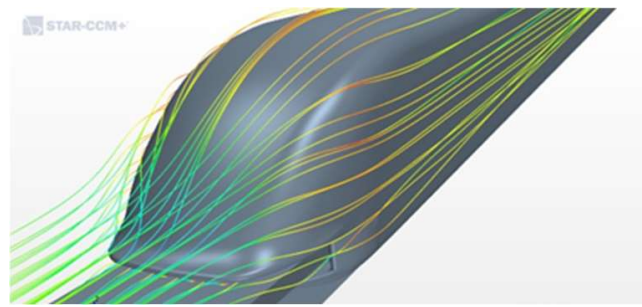

(5) $\mathrm{D}$

Fig. 10. Optimization model head streamline diagram. 
2. The decrease of high-pressure area near the stagnation point leads to the improvement of aerodynamic drag. The most effective aerodynamic drag reduction is $4.8 \%$. The decrease of aerodynamic drag in deformation zones mainly comes from the decrease of the pressure drag. The deformation of the nose tip leads to the acceleration of air velocity around the nose tip, which reduces the aerodynamic resistance.

3. The sensitive area of the car body is the nose tip, and the deformation in this area can achieve the best aerodynamic drag reduction. Through the closed-loop optimization of two adjoint methods, this information is consistent with the aerodynamic performance changes given by the optimization.

4. In the optimization execution, we chose a relatively small step deformation factor to prevent the geometric surface from being generated due to no dimensional constraints and aesthetic constraints. It is necessary to add some geometric constraints in the future research to get as close as possible to the best candidate of the objective function. It was also observed that the quality of deformation mesh generation decreased while the closed-loop optimization was carried out. Therefore, the closed-loop adjoint optimization has a certain number of optimization times in this study.

\section{ACKNOWLEDGEMENTS}

The authors would like to acknowledge the financial support from the National Key R\&D Program of China (Grant number 2016YFB1200602-11, 2016YFB1200602-12) and the Fundamental Research Funds for the Central Universities of Cenral South University.

\section{REFERENCES}

Baker, C. (2010). The flow around high speed trains. Journal of Wind Engineering and Industrial Aerodynamics 98(6-7), 277-298, SI.

Bobrowski, K., E. Ferrer, E. Valero and H. Barnewitz (2017). Aerodynamic Shape Optimization Using Geometry Surrogates and Adjoint Method. AIAA Journal 55(10), 33043317.

Giles, M. and N. Pierce (1997). Adjoint equations in CFD: duality, boundary conditions and solution behaviour. In AIAA Paper 1997-1850 13th fluid dynamics conference, New Orleans, LA.

Han, T., S. Kaushik, K. Karbon, B. Leroy, K. Mooney, S. Petropoulou and J. Papper (2016). Adjoint-drivenaerodynamic shape optimization based on a combination of steady state and transient flow solutions. SAE Int J Passenger Cars - Mech Syst 9(2), 695-709.

Hemida, H. and S. Krajnovic (2010). LES study of the influence of the nose shape and yaw angles on flow structures around trains. Journal of Wind Engineering and Industrial Aerodynamics 98, 34-46.

Huang, S., Z .W. Li and M. Z. Yang (2019) Aerodynamics of high-speed maglev trains passing each other in open air. Journal of Wind Engineering and Industrial Aerodynamics 188, 151-160.

Jameson, A. (1988). Aerodynamic Design via Control Theory. Journal of Scientific Computing 3(3), 233-260.

Jameson, A. and L. Martinelli (1998). Optimum Aerodynamic Design using the Navier-Stokes Equations. Theoretical and Computational Fluid Dynamics 10, 213-237.

Jia, Y. X. and Y. G. Mei (2018). Numerical simulation of pressure waves induced by highspeed maglev trains passing through tunnels. International Journal of Heat and Technology 36(2), 687-696.

Kapsoulis, D., K. Tsiakas, X. Trompoukis, V. Asouti and K. Giannakoglou (2016). A PCA-assisted hybrid algorithm combining EAs and adjoint methods for CFD-based optimization. Applied Soft Computing 73, 520-529

Krajnovic, S., P. Ringqvist, K. Nakade and B. Basara (2012). Large eddy simulation of the flow around a simplified train moving through a crosswind flow. Journal of Wind Engineering and Industrial Aerodynamics 110, 86-99.

Kubota, M., S. Tokuda and Y. Noguchi (2016). Development of CFDinverse analysis technology using the transient adjoint methodand its application to engine in-cylinder flow. SAE Int J Engines 9(2), 675-683.

Kungurtsev, P. V. and M. P. Juniper (2019). Adjointbased shape optimization of the microchannels in an inkjet printhead. Journal of Fluid Mechanics 871, 113-138.

Lei, R. W., J. Q. Bai and D. Y. Xu (2019). Aerodynamic optimization of civil aircraft with wing-mounted engine jet based on adjoint method. Aerospace Science and Technology 93.

Li, R., P. Xu, Y. Peng and P. Ji (2016). Multiobjective optimization of a high-speed train head based on the FFD method. Journal of Wind Engineering and Industrial Aerodynamics 152, 41-49.

Li, R., P. Xu and S. G. Yao (2020). Optimization of the high-speed train head using the radial basis function morphing method. Proceedings of The Institution of Mechanical Engineers Part FJournal of Rail and Rapid Transit 234(1), 96107.

Mueller, L., Z. Alsalihi and T. Verstraete (2012). Multidisciplinary optimization of a turbocharger radial turbine. ASME Journal of Turbomachine, 135(2), 021022-1, 021022-9.

Munoz-Paniagua, J., J. Garcia and A. Crespo (2014). 
H. C. Li et al. / JAFM, Vol. 14, No. 6, pp. 1839-1850, 2021.

Genetically aerodynamic optimization of the nose shape of a high-speed train entering a tunnel. Journal of Wind Engineering and Industrial Aerodynamics 130, 48-61.

Munoz-Paniagua, J., J. Garcia, A. Crespo and F. Laspougeas (2015). Aerodynamic Optimization of the Nose Shape of a Train Using the Adjoint Method. Journal of Applied Fluid Mechanics 8(3), 601-612.

Nejati, A. and K. Mazaheri (2017). Application of the adjoint optimization of shock control bump for ONERA-M6 wing. European Journal of Computational Mechanics 2(5-6), 557-583.

Othmer, C. (2014) Adjoint methods for car aerodynamics. J Math Ind 4(6), 1-23.

Othmer, C. and T. Grahs (2005). Approaches to Fluid Dynamic Optimization in the Car Development Process. In 6th International Conference on Evolutionary and Deterministic Methods for Design, Optimization and Control with Applications to Industrial and Societal Problems, EUROGEN 2005, Munich, Germany.

Papoutsis-Kiachagias, E. M. and K. C. Giannakoglou (2016). Continuous adjoint methods for turbulent flows, applied to shape and topology optimization: industrial applications. Arch Comput Methods Engineering 2(2), 255-299.

Papoutsis-Kiachagias, E. M., V. G. Asouti, K. C. Giannakoglou, K. Gkagkas, S. Shimokawa and E. Itakura (2019). Multi-point aerodynamic shape optimization of cars based on continuous adjoint. Structural and Multidisciplinary Optimization 59(2), 675-694.
Papadimitriou, D. I. and K. C. Giannakoglou (2008). Aerodynamic Shape Optimization Using First and Second Order Adjoint and Direct Approaches. Archives of Computational Methods in Engineering. 15(4), 447-488.

Pironneau, O. (1974). On Optimum Design in Fluid Mechanics. Journal of Fluid Mechanics, 64, 97-110.

Verma, I., C. Hill and M. Xu (2018). Multi-objective adjoint optimization of flow-bench port geometry. In: SAE WCX world congress experience, 2018-01-0772.

Vytla, V. V. (2011). Multidisciplinary Optimization Framework for High Speed Train using Robust Hybrid GA-PSO Algorithm. Ph. D. Thesis, Wright State University.

Wang, K., S. J. Yu and Z. Wang (2018). Adjointbased airfoil optimization with adaptive isogeometricdiscontinuous Galerkin method. Applied Mechanics and Engineering 344, 602625.

Yao, S. B., D. L. Guo, Z. X. Sun, G. W. Yang and D. W. Chen (2014).Optimization design for aerodynamic elements of high speed trains. Computers \& Fluids 95, 56-73.

Zhang, L., J. Y. Zhang, T. Li and Y. D. Zhang (2017). Multi-objective aerodynamic optimization design of high-speed train head shape. Journal of Zhejiang University-Science A 18(11), 841-854.

Zhang, L. (2017). Study on Aerodynamic Shape Optimization Design of High-Speed Trains. Ph. D. Thesis, Southwest Jiaotong University. 